\title{
High prevalence of rare FBLIM1 gene variants in an Italian cohort of patients with Chronic Non-bacterial Osteomyelitis (CNO)
}

\author{
Adamo Pio d'Adamo 1,2, Anna Monica Bianco², Giovanna Ferrara', Martina La Bianca², Antonella Insalaco ${ }^{3}$, \\ Alberto Tommasini ${ }^{2}$, Manuela Pardeo ${ }^{3}$, Marco Cattalini ${ }^{4}$, Francesco La Torre ${ }^{5}$, Martina Finetti ${ }^{6}$, Clotilde Alizzi ${ }^{7}$, \\ Gabriele Simonini ${ }^{8}$, Virginia Messia ${ }^{3}$, Serena Pastore ${ }^{2}$, Rolando Cimaz ${ }^{9}$, Marco Gattorno ${ }^{6}$, Andrea Taddio ${ }^{1,2^{*}}$ (D) \\ and for the Italian Pediatric Rheumatology Study Group
}

\begin{abstract}
Background: FBLIM1 gene has been recently demonstrated to be involved in the pathogenesis of bone sterile inflammation. The aim of the study is to evaluate the prevalence of FBLIMI gene variants in a cohort of 80 Italian patients with Chronic Non-bacterial Osteomyelitis (CNO).

Methods: The coding regions of FBLIM1 gene were sequenced in a cohort of 80 patients with CNO using DNA extracted from blood lymphocytes, and PCR products were sequenced. Only rare (global MAF $<2 \%$ ), coding variants detected were considered. Clinical evaluation of patients with rare variants and those without was performed. Fisher's exact test was used to compare categorical and ordinal data, and Student's t-test was used to analyze continuous data.
\end{abstract}

Results: Eighteen out of 80 patients $(\sim 22 \%)$ presented at least one rare coding variant in FBLIM1. Eight patients presented a variant never associated before with CNO. All patients presented classical features of CNO and no statistical difference between patients with presence of FBLMII variants and those without were found in terms of clinical manifestation, treatment, and outcome.

Conclusion: Considering the high frequency of rare variants in our CNO cohort, our data seem to confirm a possible role of FBLIM1 in the pathogenesis of CNO suggesting that $C N O$ is a disorder of chronic inflammation and imbalanced bone remodeling.

Keywords: Chronic non-bacterial osteomyelitis, FBLIM1 gene, Bone sterile inflammation, Autoinflammatory disease

\section{Background}

Chronic Non-bacterial Osteomyelitis $(\mathrm{CNO})$ is a rare inflammatory disorder that is characterized by onset of pain, local bone expansion and radiological findings suggestive of osteomyelitis, usually at multiple sites, not

\footnotetext{
* Correspondence: andrea.taddio@burlo.trieste.it

${ }^{1}$ University of Trieste, Trieste, Italy

${ }^{2}$ Institute for Maternal and Child Health - IRCCS "Burlo Garofolo", Via dell'Istria 65/1, 34100 Trieste, Italy

Full list of author information is available at the end of the article
}

related to an infectious disease [1]. Although its pathogenesis remains still unknown, there is consensus about the hypothesis that $\mathrm{CNO}$ might be a genetic disease within the spectrum of autoinflammatory disorders [2-4]. Although the existence of genes contributing to sporadic $\mathrm{CNO}$ has been proposed, their identification is still missing [5].

Cox et al have recently demonstrated that FBLIM1, a gene that codes for a protein involved in the regulation of bone remodeling, could be involved in the 
pathogenesis of sterile bone inflammation. The authors, via whole-exome sequencing, detected a homozygous mutation in the filamin-binding domain of FBLIM1 in an affected child with consanguineous parents. They also sequenced FBLIM1 in 96 subjects with $\mathrm{CNO}$ and found a second patient with a distinct frameshift variant [6].

On this basis, we sequenced the FBLIM1 gene in a cohort of Italian patients with $\mathrm{CNO}$ and correlated the results with clinical manifestations.

\section{Methods}

This is a multicenter observational study. In the absence of validated international diagnostic criteria, we used the proposed Jansson criteria for $\mathrm{CNO}$ diagnosis [7]. The clinical and radiological data were entered into a customized and anonymized database and considered as variables for correlation analysis. Blood samples were collected from patients affected by $\mathrm{CNO}$ from 7 Italian rheumatology centers: IRCCS Burlo Garofolo, Trieste; Spedali Civili, Brescia; Anna Meyer Children's Hospital, Florence; Bambino Gesù Children's Hospital, Rome; G. Di Cristina Children's Hospital, Palermo; "Giovanni XXIII" Pediatric Hospital, Bari and IRCCS Giannina Gaslini, Genoa. Written informed parental consent, according to the approved protocol of the IRCCS "Burlo Garofolo" ethics committee ( $\left.{ }^{\circ} 27 / 14\right)$ was obtained for genetic analysis. Blood collected for DNA analysis was taken specifically for this study during a routine blood sampling. After standard genomic isolation, DNA was used for Sanger sequencing on entire coding and flanking regions of FBLIM1. All sequences were analyzed with codon code aligner software (V.7.1.1 version). Only rare (global $\mathrm{MAF}<2 \%$ ), coding variants detected were considered. To predict if each variation could be harmful for the protein function, we used the following softwares: Polyphen2 (http://genetics.bwh.harvard.edu/pph2/ ); SIFT (http://sift.jcvi.org); MUTATION ASSESSOR (http://mutationassessor.org/r3/); Human Splicing Finder (http://www.umd.be/HSF/) and TRANSFAC (http://gene-regulation.com/pub/databases.html\#transfac). Controls were taken from the gnomAD, a worldwide database of hundreds of thousands of individuals with no manifested pathologies, stratified by regions of origin.

Clinical evaluation between patients with and without rare variants was performed. Fisher's exact test was used to compare categorical and ordinal data, and Student's t test was used to analyze continuous data.

\section{Results}

Eighty patients diagnosed with $\mathrm{CNO}$ were enrolled, 52 females (65\%) and 28 males (35\%). Bone pain was the most common clinical complaint; median age at onset was 9 years (range 4-14 years). We found a median number of six bone localizations per patient; 9 patients had a single lesion. Most lesions were located to the long bones (65\%), pelvis (48\%) and spine (37\%). Three patients presented skull lesions (they underwent bone biopsy to exclude malignancy). Five patients (5.8\%) had a first-degree relative affected by an autoimmune disorder. Thirty patients (35\%) had a comorbidity; among these, 18 (22.5\%) had skin manifestations such as psoriasis, pustulosis or severe acne, 4 (5\%) had inflammatory bowel disease. No consanguinity nor family history for $\mathrm{CNO}$ was reported.

Sanger sequencing was conducted in all patients. 18 out of $80(22,5 \%)$ patients presented at least one rare (s 0.02 ) variant in FBLIM1 gene. In particular, 15 patients presented 1 rare variant; 2 patients presented 2 rare variants and 1 patient had 5 rare variants. In total, 11 rare variants were found ( 7 out of 11 variants presented a $\operatorname{MAF} \leq 0.01)$.

Table 1 displays the variants /SNPs identified. 5 out of 11 variants were in the coding regions and 6 were intronic. Among the SNP in the coding region 3 are missense and 2 synonymous variants. Two of the missense (Arg38Gln and Gly311Arg) and one of the intronic variants (c. $250+32 \mathrm{C}>\mathrm{A}$ ) have been previously reported in $\mathrm{CNO}$ by $\mathrm{Cox}$ et al [9]. The other eight variants have never been associated with $\mathrm{CNO}$.

Patient P18 harbored 5 FBLIM1 variants: two missense variants (rs146575757 Arg38Gln and rs114077715 Gly311Arg), two intronic (rs201006671 and rs76050903) and one synonymous (rs61733331) variant. By parental sequencing we verified that the child inherited from his mother the allele with the missense Arg38Gln, the two intronic and the synonymous variant while he inherited from his father the allele with the only Gly311Arg missense variant, found also in heterozygosis in P16. In patient P13 we identified in homozygosis the intronic variant rs187479896 and in heterozygosis the rs140170023, a synonymous variant present also in heterozygosis in other 9 patients (P2, P3, P4, P5, P6, P8, P9, $\mathrm{P} 10, \mathrm{P} 13$ and $\mathrm{P} 15)$. In patient $\mathrm{P} 3$, in addition to the synonymous variant, the SNPs rs41310367 was also detected in heterozygosis. An intronic heterozygous variant was identified also in P11. Finally, P12 carried the missense variant Arg22His (rs540511146), P7 the variant rs766409425 and patients P1, P14, and P17 the intronic variant rs144567113.

All the 11 identified variants, especially those never described before and so orphaned of functional studies were analyzed "in silico" using specific software; results are shown in Table 2. Moreover, according to dbSNP, all the variants identified are extremely rare in healthy controls examined (supplementary table), thus enforcing their possible pathogenic roles. 
Table 1 Rare variants of FBLIM1 gene identified in 18 patients affected by Chronic Non-Bacterial Osteomyelitis

\begin{tabular}{|c|c|c|c|c|c|c|}
\hline & rs & Change & Amino acid changes & MAF & Variant & Patients \\
\hline 1 & rs540511146 & c. $65 G>A$ & p.Arg22His & $A=0.00011$ & missense & P12 \\
\hline 2 & rs201006671 & c. $-20-49 \mathrm{G}>\mathrm{T}$ & N/A & $T=0.0033$ & intron & P18 \\
\hline 3 & rs76050903 & C. $-20-48 C>A$ & N/A & $A=0.0033$ & intron & P18 \\
\hline 4 & rs146575757 ${ }^{\mathrm{a}}$ & C. $113 \mathrm{G}>\mathrm{A}$ & p.Arg38Gln & $A=0.00391$ & missense & P18 \\
\hline 5 & rs61733331 & c. $222 \mathrm{G}>\mathrm{A}$ & p.Pro74= & $A=0.00675$ & synonymous & P18 \\
\hline 6 & rs766409425 & $c .541+13 G>A$ & N/A & $A=0.00003$ & intron & P7 \\
\hline 7 & rs187479896 & c. $717+14 A>G$ & N/A & $G=0.00083$ & intron & P13 \\
\hline 8 & rs41310367 ${ }^{a}$ & $c .250+32 C>A$ & N/A & $\mathrm{T}=0.0191$ & intron & P3; P11 \\
\hline 9 & rs140170023 & $c .447 G>A$ & p.Ala149= & $A=0.01200$ & synonymous & $\mathrm{P} 2, \mathrm{P3}, \mathrm{P} 4, \mathrm{P5}, \mathrm{P} 6, \mathrm{P} 8, \mathrm{P9}, \mathrm{P10}, \mathrm{P} 13, \mathrm{P} 15$ \\
\hline 10 & rs114077715 ${ }^{a}$ & $c .931 G>A$ & p.Gly311Arg & $A=0.01955$ & missense & P18; P16 \\
\hline 11 & rs144567113 & c.718-29C > T & N/A & $T=0.01515$ & intron & P1; P14; P17 \\
\hline
\end{tabular}

${ }^{a}$ variants already described [8]

All patients presented classical features of $\mathrm{CNO}$ and no statistical differences between patients with or without FBLIM1 variants were found in terms of gender prevalence, positive family history, age at onset, number of sites involved, presence of fever, arthritis and skin involvement as well as remission at the end of follow-up (Table 3).

\section{Discussion}

$\mathrm{CNO}$ is a rare pediatric autoinflammatory bone disease and several factors such as family pedigree and syndromic monogenic forms of $\mathrm{CNO}$ suggest a strong genetic component. Moreover, the association between the presence of polymorphisms of the IL-10 promoter with $\mathrm{CNO}$ pathogenesis has been reported [10]. It has been also demonstrated that mutation of the pstpip 2 gene in mice results in an autoinflammatory disease very similar to human $\mathrm{CNO}$ [8]. However, candidate genes including PSTPIP1, CARD15/NOD2, and IL1RN, were not associated with $\mathrm{CNO}$ in humans when analyzed in small cohorts $[11,12]$.

Cox et al. recently showed that recessive mutations in FBLIM1 contribute to the pathogenesis of CNO [9]. FBLIM1 codes for Filamin-binding LIM protein 1

Table 2 The in-silico analysis of the 11 FBLIM1 gene variants

\begin{tabular}{|c|c|c|c|c|c|}
\hline \multicolumn{2}{|l|}{ NonSynonymous } & \multirow{2}{*}{$\begin{array}{l}\text { Global MAF } \\
\text { Allele/MAF }\end{array}$} & \multicolumn{3}{|l|}{ In Silico Analysis } \\
\hline rs & CDS/AA & & PP2 & SIFT CADD & Mutation Assessor \\
\hline rs540511146 & c. $65 \mathrm{G}>\mathrm{A} ; \mathrm{p} . \mathrm{R} 22 \mathrm{H}$ & $\mathrm{A} / 0.00011$ & PD & $D \perp B$ & Medium \\
\hline rs146575757 & c.113G > A; p. R38Q & A/0.00391 & B & TB & Low \\
\hline rs114077715 & c.931G > A; p. G311R & A/0.01955 & B & $D \perp B$ & No, data \\
\hline \multicolumn{2}{|l|}{ Synonymous } & Global MAF & \multicolumn{3}{|c|}{ In Silico Analysis (HSF) } \\
\hline rs & CDS/AA & Allele/MAF & ESS & ESE & splicing site \\
\hline rs61733331 & c.222G > A; p. Pro74Pro & $\mathrm{A} / 0.00675$ & New ESS Site & ESE site broken & NASS \\
\hline rs140170023 & c.447G > A; p.Ala149Ala & A/0.01200 & New ESS Site & & NASS \\
\hline \multicolumn{2}{|l|}{ Intronic Variants } & Global MAF & In silico analysis & & \\
\hline rs & SNVs & Allele/MAF & New Site & Broken Site & TRANSFAC \\
\hline rs201006671 & c. $-20-49 G>T$ & $\mathrm{~T} / 0.0033$ & & & Broken site for ENKTF-1 \\
\hline rs76050903 & C. $-20-48 C>A$ & A/0.0033 & & & Broken site for ENKTF-1 \\
\hline rs766409425 & $c .541+13 G>A$ & A/0.00003 & SF2/ASF & SRp40 & \\
\hline rs187479896 & c. $717+14 \mathrm{~A}>\mathrm{G}$ & $\mathrm{G} / 0.00083$ & & & \\
\hline rs41310367 & c. $250+32 C>A$ & $\mathrm{~T} / 0.0191$ & & SF2/ASF/SRp55 & \\
\hline rs144567113 & c.718-29C > T & $\mathrm{T} / 0.01515$ & & & \\
\hline
\end{tabular}

Reference SNPs: rs; coding sequence/aminoacids: CDS/AA; Polyphen-2: PP2

Sorting Intolerant from Tolerant: SIFT; Combined Annotation Dependent Depletion: CADD

Probably Damaging: PD; Deleterious: D; Benign: B; Tolerated: T

Minor Allele Frequency: MAF; Exonic Splicing Silencer: ESS; Exonic Splicing Enhancer: ESE 
Table 3 Clinical presentation, laboratory data, treatment and outcome of CNO patients cohort divided following presence or absence of FBLIM1 variant. § 9 patients were lost at last follow-up

\begin{tabular}{|c|c|c|c|}
\hline & Patients without FBLIM1 variants (62 patients) & Patients with FBLIM1 variants (18 patients) & $P$ \\
\hline Gender female & $41(66 \%)$ & $11(61 \%)$ & 0,78 \\
\hline Median Age at onset & 9.5 y (range $1.5-16)$ & 8.9 y (range $1,5-12$ ) & 0,97 \\
\hline \multicolumn{4}{|l|}{ Clinical data } \\
\hline Fever & $30(48 \%)$ & $4(22 \%)$ & 0,06 \\
\hline Swelling & $11(18 \%)$ & 7 (38\%) & 0,1 \\
\hline Pain & $60(96 \%)$ & $15(83 \%)$ & 0,07 \\
\hline Sites & & 8.9 (median) & \\
\hline Long bones & $46(74 \%)$ & $13(72 \%)$ & 1,0 \\
\hline Pelvis & $23(37 \%)$ & $11(61 \%)$ & 0,1 \\
\hline Column & $22(35 \%)$ & $5(27 \%)$ & 0,78 \\
\hline Clavicle & $16(26 \%)$ & $5(27 \%)$ & 1,0 \\
\hline Chest and ribs & $24(39 \%)$ & $3(16 \%)$ & 0,09 \\
\hline Foot and hands & $15(24 \%)$ & $4(22 \%)$ & 1,0 \\
\hline Mandible & $11(18 \%)$ & $1(5 \%)$ & 0,28 \\
\hline Skulls & $3(5 \%)$ & $0(0 \%)$ & 1,0 \\
\hline Skin involvements & $11(18 \%)$ & $4(22 \%)$ & 0,73 \\
\hline Bowel involvements & $3(5 \%)$ & $1(6 \%)$ & 1,0 \\
\hline Renal involvements & $2(3 \%)$ & $0(0 \%)$ & 1,0 \\
\hline \multicolumn{4}{|l|}{ Laboratory data } \\
\hline Elevated CRP & $32(52 \%)$ & $14(77 \%)$ & 0,06 \\
\hline Elevated ESR & $41(66 \%)$ & $13(72 \%)$ & 0,78 \\
\hline \multicolumn{4}{|l|}{ Treatment } \\
\hline NSAIDs & 55 (89\%) & 17 (94\%) & 0,67 \\
\hline Steroids & $20(32 \%)$ & $6(33 \%)$ & 1,0 \\
\hline MTX & 12 (19\%) & $3(16 \%)$ & 1,0 \\
\hline Sulfasalazine & $10(16 \%)$ & $3(16 \%)$ & 1,0 \\
\hline Adalimumab & $3(5 \%)$ & $1(5 \%)$ & 1,0 \\
\hline Etanercept & $8(13 \%)$ & $2(11 \%)$ & 1,0 \\
\hline Infliximab & $3(5 \%)$ & $2(11 \%)$ & 0,31 \\
\hline Anakinra & $9(15 \%)$ & $3(17 \%)$ & 1,0 \\
\hline Neridronate & $2(3 \%)$ & $1(6 \%)$ & 1,0 \\
\hline Pamidronate & $29(47 \%)$ & $8(44 \%)$ & 1,0 \\
\hline \multicolumn{4}{|l|}{ Outcome at last follow up§ } \\
\hline Remission without therapy & $32(52 \%)$ & $5(28 \%)$ & 0,11 \\
\hline Remission on therapy & $18(29 \%)$ & $5(28 \%)$ & 1,0 \\
\hline Active disease & $6(10 \%)$ & $5(28 \%)$ & 0,11 \\
\hline
\end{tabular}

(FBLP1 or migfilin), a filamin-binding protein involved in the regulation of bone remodeling [13].

FBLP1 is a key regulator of the cytoskeleton, as it is recruited to cell-matrix contacts in response to adhesion and colocalizes with beta-catenin at cell-cell junctions in epithelial and endothelial cells. Through interactions with multiple binding partners, including filamin, FBLP1 links the cell adhesion structures to the actin cytoskeleton [14].
FBLP1 competes with integrin $\beta$ for filamin binding to promote integrin activation in neutrophils as well as bone homeostasis [15]. Therefore, a mutation in the filaminbinding domain of FBLP1 may disrupt FBLP1-FLN binding, resulting in aberrant integrin activation in neutrophils and leading to sterile inflammation.

In our cohort of $80 \mathrm{CNO}$ patients we found a rare coding variant of FBLIM1 in 18 patients. Three of these 
variants (rs146575757, rs41310367 and rs114077715) were previously described by Cox et al. [6]. The missense variant Arg38Gln (rs146575757) is in the filaminbinding domain (exon 3), so it may be causative of neutrophil activation. The second variant (rs41310367) is centrally located in the middle of an enhancer, in a STAT3 binding region and in an NR4A2 recognition site, reported to be active in several cell lines, including osteoblasts, so it may disrupt balance between osteoclasts and osteoblasts activity leading to bone remodeling. The other missense variant Gly311Arg on the last exon is localized in the third LIM domain of the protein, a small protein -protein interaction domain, containing two zinc fingers.

For the further eight variants identified in our study no literature data are available about their association with the disease or their functional impact. These variants are all rare $(\mathrm{MAF}<2 \%)$ in the general population, and extremely rare in the case of rs540511146 (MAF = 0.00011 ). The high prevalence of FBLM1 rare variants in our $\mathrm{CNO}$ cohort may support a role in the pathogenesis of the disease.

Considering the intronic variants, they were predicted to possibly alter binding sites for splicing factors, in particular for the serine/arginine-rich protein (SR) specific binding sites. We observed by Human Splicing Finder a prediction in which the substitution of a single nucleotide in that specific genomic region leads to a loss and/ or possible new sequence recognition by these factors and a possible creation a sequence for a new splice site. The in silico analysis and its predictions could address the research towards functional studies to verify the pathogenicity of the variants.

No statistical association was found between patients with and without FBLIM1 gene variants, suggesting that FBLIM1 might be considered a non-specific predisposing factor to $\mathrm{CNO}$ in a subgroup of patients.

\section{Conclusions}

$\mathrm{CNO}$ remains a not completely understood disease but it probably belongs to the family of autoinflammatory diseases. A unique causing gene was not found yet. However, our data seem may support the fact that some FBLIM1 variants might increase the susceptibility to the $\mathrm{CNO}$ pathogenesis.

\section{Supplementary information}

Supplementary information accompanies this paper at https://doi.org/10. 1186/s12969-020-00447-4.

Additional file 1: Supplementary table. Frequencies (Global, European, South Asian, East Asian and African) of FBLIM1 gene variants in healthy control people (from GnomAD v2.1.1 controls dataset).

\section{Abbreviation}

CNO: Chronic Non-bacterial Osteomyelitis

\section{Acknowledgements}

Not applicable.

\section{Authors' contributions}

Andrea Taddio, Adamo Pio d'Adamo, Giovanna Ferrara, Rolando Cimaz and Marco Gattorno: conceptualized and designed the study, drafted the initial manuscript, and approved the final manuscript as submitted. Anna Monica Bianco and Martina La Bianca performed the Sanger analysis and the evaluation of the variants. Serena Pastore, Alberto Tommasini, Marco Cattalini, Gabriele Simonini, Virginia Messia, Antonella Insalaco, Clotilde Alizzi, Manuela Pardeo, Martina Finetti and Francesco La Torre carried out the initial analyses, reviewed and revised the manuscript, and approved the final manuscript as submitted. All authors approved the final manuscript as submitted and agree to be accountable for all aspects of the work.

\section{Funding}

The manuscript was supported by Institute for Maternal and Child Health IRCCS "Burlo Garofolo" (grant number RC 27/14).

\section{Availability of data and materials}

The datasets used and/or analysed during the current study are available from the corresponding author on reasonable request.

Ethics approval and consent to participate

The study protocol and genetic tests were approved by IRCCS "Burlo Garofolo" ethics committee ( $\left.n^{\circ} 27 / 14\right)$.

Consent for publication

Not applicable.

\section{Competing interests}

The authors declare that they have no competing interests.

\section{Author details}

${ }^{1}$ University of Trieste, Trieste, Italy. ${ }^{2}$ Institute for Maternal and Child Health IRCCS "Burlo Garofolo", Via dell'Istria 65/1, 34100 Trieste, Italy. ${ }^{3}$ Department of Pediatric Medicine, Division of Rheumatology, Bambino Gesù Children's Hospital, Rome, Italy. ${ }^{4}$ Pediatric Clinic University of Brescia and Spedali Civili of Brescia, Brescia, Italy. ${ }^{5}$ Pediatric Rheumatology Center, Pediatric Unit, "Giovanni XXIII", Pediatric Hospital, Bari, Puglia, Italy. ${ }^{6}$ Centro Malattie Autoinfiammatorie e Immunodeficenze, IRCCS "G. Gaslini", Genoa, Italy. " "G. Di Cristina"Children's Hospital, Palermo, Italy. ${ }^{8}$ Pediatric Rheumatology Unit, AOU Meyer, University of Florence, Florence, Italy. ${ }^{9}$ Azienda Socio Sanitaria Territoriale (ASST) G.Pini and University of Milan, Milan, Italy.

Received: 25 November 2019 Accepted: 3 July 2020 Published online: 10 July 2020

References

1. Taddio A, Ferrara G, Insalaco A, Pardeo M, Gregori M, Finetti M, et al. Dealing with Chronic Non-Bacterial Osteomyelitis: a practical approach. Pediatr Rheumatol Online J. 2017:15:87.

2. Majeed HA, Kalaawi M, Mohanty D, Teebi AS, Tunjekar MF, al-Gharbawy F, et al. Congenital dyserythropoietic anemia and chronic recurrent multifocal osteomyelitis in three related children and the association with sweet syndrome in two siblings. J Pediatr. 1989:115:730-4.

3. Caorsi R, Picco P, Buoncompagni A, Martini A, Gattorno M. Osteolytic lesion in PAPA syndrome responding to anti-interleukin 1 treatment. J Rheumatol. 2014:41:2333-4

4. Aksentijevich I, Masters SL, Ferguson PJ, Dancey P, Frenkel J, van RoyenKerkhoff A, et al. An autoinflammatory disease with deficiency of the interleukin-1-receptor antagonist. N Engl J Med. 2009;360:2426-37.

5. Golla A, Jansson A, Ramser J, Hellebrand H, Zahn R, Meitinger T, et al. Chronic recurrent multifocal osteomyelitis (CRMO): evidence for a susceptibility gene located on chromosome 18q21.3-18q22. Eur J Hum Genet. 2002;10:217-21.

6. Cox AJ, Darbro BW, Laxer RM, Velez G, Bing X, Finer AL, et al. Recessive coding and regulatory mutations in FBLIMI underlie the pathogenesis of 
chronic recurrent multifocal osteomyelitis (CRMO). PLoS One. 2017;12: e0169687.

7. Jansson AF, Muller TH, Gliera L, Ankerst DP, Wintergest U, Belohradsky BH, et al. Clinical score for nonbacterial osteitis in children and adults. Arthritis Rheum. 2009;60:1152-9.

8. Ferguson PJ, Bing X, Vasef MA, Ochoa LA, Mahgoub A, Waldschmidt TJ, et al. A missense mutation in pstpip2 is associated with the murine autoinflammatory disorder chronic multifocal osteomyelitis. Bone. 2006;38: $41-7$.

9. Cox AJ, Ferguson PJ. Update on the genetics of nonbacterial osteomyelitis in humans. Curr Opin Rheumatol. 2018;30:521-5.

10. Hamel J, Paul D, Gahr M, Hedrich CM. Pilot study: possible association of IL10 promoter polymorphisms with CRMO. Rheumatol Int. 2012:32:555-6.

11. Beck C, Girschick HJ, Morbach H, Schwarz T, Yimam T, Frenkel J, et al. Mutation screening of the IL-1 receptor antagonist gene in chronic nonbacterial osteomyelitis of childhood and adolescence. Clin Exp Rheumatol. 2011:29:1040-3.

12. Huber AM, Lam PY, Duffy CM, Yeung RS, Ditchfield M, Laxer D, et al. Chronic recurrent multifocal osteomyelitis: clinical outcomes after more than five years of follow-up. J Pediatr. 2002;141:198-203.

13. Xiao G, Cheng H, Cao H, Chen K, Tu Y, Yu S, et al. Critical role of filaminbinding LIM protein 1 (FBLP-1)/migfilin in regulation of bone remodeling. J Biol Chem. 2012;287:21450-60.

14. Wu C. Migfilin and its binding partners: from cell biology to human diseases. J Cell Sci. 2005;118:659-64.

15. Das M, Ithychanda SS, Qin J, Plow EF. Migfilin and filamin as regulators of integrin activation in endothelial cells and neutrophils. PLoS One. 2011;6: e26355.

\section{Publisher's Note}

Springer Nature remains neutral with regard to jurisdictional claims in published maps and institutional affiliations.

Ready to submit your research? Choose BMC and benefit from:

- fast, convenient online submission

- thorough peer review by experienced researchers in your field

- rapid publication on acceptance

- support for research data, including large and complex data types

- gold Open Access which fosters wider collaboration and increased citations

- maximum visibility for your research: over $100 \mathrm{M}$ website views per year

At BMC, research is always in progress.

Learn more biomedcentral.com/submissions 